
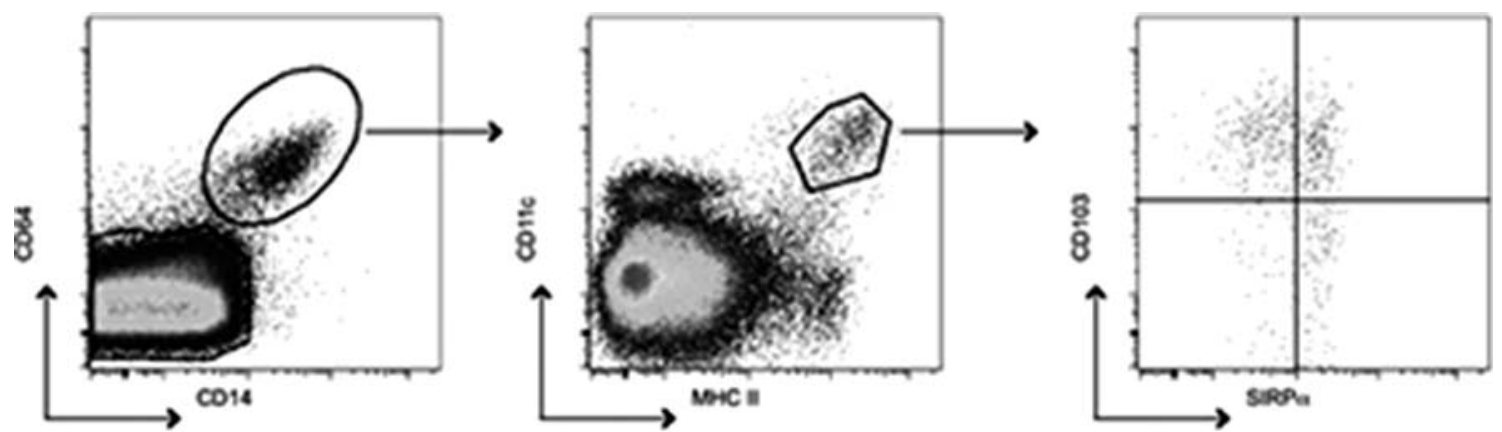

Abstract PTU-077 Figure 1 Gating strategy to identify three subsets of dendritic cells in a colonic biopsies from an individual unaffected by IBD.

Conclusion Our novel multi-parameter analyses of live cells prepared from fresh colonic and ileal biopsies enable precise examination of the disease-inducing and effector populations that drive UC and CD. We are using the methods described here to dissect the immunological mechanisms driving inflammation in patients with IBD. We anticipate that our on-going comparisons of each of these immune cell populations in blood and intestinal biopsies from unaffected individuals, CD, and UC patients will reveal important details about pathogenic mechanisms controlling intestinal inflammation.

Disclosure of Interest None Declared.

\section{PTU-078 THE APPLICATION OF A MARKOV MODEL FOR EVAUATING ASA THERAPY FOR ULCERATIVE COLITIS}

${ }^{1}$ V Mukhekat, ${ }^{2}$ A Ahmad, ${ }^{1} Y$ Merali, ${ }^{2} D$ Aldulaimi*. 'Warwick Business School, University of Warwick, Coventry, UK; ${ }^{2}$ General Medicine, Worcestershire Acute Hospitals NHS Trust, Worcestershire, UK

\subsection{6/gutjnl-2014-307263.152}

Introduction We developed a Markov model for ulcerative colitis that calculated the costs of two different treatment strategies for 1000 patients and healthcare providers and ran the model over a ten year cycle to evaluate each treatment arm.

Methods Healthcare costs were calculated from the published study of costs of ulcerative colitis at Aintree University Hospital, Liverpool, UK. Effects were quantified with the EQ-5D visual analogue scale and Work Productivity and Activity Impairment (WPAI) study.

Lost income for patients was calculated assuming that the patient was self employed, earning the national average wage and had no sickness benefits.

Results Cohort simulation is used to present the results. At the end of exercise, 277 patients were in the phase of remission in ASA continuing arm and 232 patients were in the same phase in ASA discontinuing arm.

Total costs to the healthcare providers for providing treatment to the patients were found to be $£ 15,390,139.81$ (discounted- $£ 12,825,018.45$ ) for ASA continuing arm and that for ASA discontinuing arm were $£ 12,911,557.43$ (discounted$£ 10,752,633.35)$. These costs were incurred to gain QALYs of 31,682.14 (discounted-26713.79) for the ASA continuing arm and of 31,307.73 (discounted-26,405.89 for ASA discontinuing arm).

Primary outcome in terms of ICER (Incremental Cost-Effectiveness Ratio) is $£ 6730.55$ per QALY (acceptable range < $£ 20,000-30,000$ ). Secondary outcomes such as Discounted total earnings lost due to illness and Discounted total earnings affected due to work impairment were found to be in favour of
ASA continuation by $19.43 \%$ ( $£ 3.7$ million $)$ and $11.15 \%$ ( $£ 7.8$ million) in favour of ASA continuing arm.

Sensitivity analysis was conducted to challenge both the cost and effect assumptions. CEAC (Cost Effectiveness Acceptability Curve) revealed the probability of 1 is reached with the costeffectiveness acceptability limit of $£ 40,000$ ceiling limit.

Conclusion Markov models have been used extensively to study the cost-effectiveness of healthcare interventions in chronic diseases.

Our Markov model considered costs for both patients and healthcare providers, rather than solely considering the costs for healthcare providers.

Results not only revealed the cost-effectiveness of ASA for the healthcare provider but also potential benefits for the broader economy.

Disclosure of Interest None Declared.

\section{PTU-079 THE TPMT AND ABCB1 POLYMORPHISMS IN IBD PATIENTS IN CRETE: IMPACT ON DISEASE AND RESPONSE TO TREATMENT}

C Coucoutsi, O Sfakianaki, G Emmanuel, E Marinidou, A Voumvouraki, I Koutroubakis, E Kouroumalis*. Gastroenterology, University of Crete Medical School, Heraklion, Greece

\subsection{6/gutjnl-2014-307263.153}

Introduction It is well known that polymorphisms of the TPMT gene (coding for thiopourine methyl-transferase), influence response to treatment with azathioprine. Polymorphisms of the ABCB1 gene (coding for p-glycoprotein 170) has been associated with IBD and resistance to treatment but results are conflicting. The aim of this study was to determine the frequencies of TPMT and ABCB1 gene polymorphisms in IBD patients from Crete, a population genetically homogeneous, and how these polymorphisms might influence response to treatment and disease behaviour.

Methods A total of 222 IBD patients records were reviewed for intake of azathioprine, possible adverse reactions, response to treatment and need for colectomy. All patients were genotyped for TPMT gene polymorphisms, that have been related to intolerance to azathioprine (G238C, G460A and A719C) as well as ABCB1 gene polymorphisms (G2677T/A and C3435T), using a PCR-RFLP method. The same polymorphisms were also determined in 119 age and sex healthy controls.

Results Allele frequencies of TPMT gene in our study population were found to be in concordance with those reported in other Caucasian populations. 76 IBD patients were identified receiving azathioprine, of whom 16 were discontinued $(10 \mathrm{CD}$, $6 \mathrm{UC})$ due to adverse reaction. 2 of them were found to carry the G460A and A719G alleles (TPMT 3A genotype) (12.5\%). 


\section{Correction}

V Mukhekat, I Ahmad, Y Merali, et al. PTU-078 The Application Of A Markov Model For Evauating Asa Therapy For Ulcerative Colitis. Gut 2014;63:Suppl 1 A73. The first author's name was published incorrectly. The correct name is Vinaykumar Mukhekar.

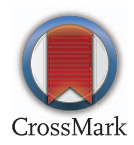

Gut 2014;63:1528. doi:10.1136/gutjnl-2014-307263.152corr1 